\title{
BMJ Open A smartphone application for reporting symptoms in adults with cystic fibrosis: protocol of a randomised controlled trial
}

\author{
Jamie Wood, ${ }^{1,2,3}$ Sue Jenkins, ${ }^{1,2,3}$ David Putrino, ${ }^{4,5}$ Siobhain Mulrennan,,6 \\ Sue Morey, ${ }^{3,6}$ Nola Cecins, ${ }^{2}$ Kylie Hill ${ }^{1,3}$
}

To cite: Wood J, Jenkins S, Putrino D, et al. A smartphone application for reporting symptoms in adults with cystic fibrosis: protocol of a randomised controlled trial. BMJ Open 2018;8:e021136. doi:10.1136/ bmjopen-2017-021136

- Prepublication history for this paper is available online. To view these files, please visit the journal online (http://dx.doi org/10.1136/bmjopen-2017021136).

Received 18 December 2017

Revised 7 March 2018

Accepted 4 April 2018

Check for updates

${ }^{1}$ School of Physiotherapy and Exercise Science, Curtin University, Perth, Western Australia, Australia

${ }^{2}$ Physiotherapy Department, Sir Charles Gairdner Hospital, Perth, Western Australia, Australia ${ }^{3}$ Institute for Respiratory Health, Sir Charles Gairdner Hospital,

Perth, Australia

${ }^{4}$ Abilities Research Center, Mount Sinai Health System, New York City, New York, USA

${ }^{5}$ Department of Rehabilitation

Medicine, Icahn School of Medicine at Mount Sinai, New York City, New York, USA

${ }^{6}$ Department of Respiratory

Medicine, Sir Charles Gairdner Hospital, Perth, Western Australia, Australia

Correspondence to

Jamie Wood;

jamie.wood@health.wa.gov.au

\section{ABSTRACT}

Introduction In people with cystic fibrosis (CF), exacerbations have been shown to have profound and prolonged negative effects such as reducing physical activity and health-related quality of life, increasing the rate of decline of lung function and healthcare costs, and ultimately increasing the risk of mortality. Delayed initiation of treatment following the signs of an exacerbation has been shown to be associated with failure to recover to baseline. Therefore, the late identification and treatment of an exacerbation due to delayed presentation will potentially worsen short-term and long-term outcomes. We have developed a smartphone application, containing questions which require yes or no responses relating to symptoms suggestive of a respiratory exacerbation. Its use is intended to facilitate the early identification of symptoms suggestive of a respiratory exacerbation, and allow the CF team to initiate treatment sooner, thereby potentially reducing the risk of severe exacerbations which require intravenous antibiotics (IVAB) and often a hospital admission.

Methods We will undertake a randomised controlled trial. 60 adults with CF will be recruited and randomised to either the intervention or control group. The intervention group will use the smartphone application weekly for 12 months, or earlier than the next weekly reporting time if they feel their symptoms have worsened. The control group will continue to receive usual care, involving regular (approximately 3 monthly) CF outpatient clinic appointments. The primary outcome measure will be courses and days of IVAB.

Ethics and dissemination Approval was obtained from the Sir Charles Gairdner Group Human Research Ethics Committee for WA Health (2015-030) and Curtin University Human Research Ethics Committee (HR212/2015), and has been registered with the Australian and New Zealand Clinical Trials Registry. Results of this study will be presented at international conferences and published in peer-reviewed journals in accordance with the Consolidated Standards of Reporting Trials statement. Trial registration number ACTRN12615000599572.

\section{INTRODUCTION}

Cystic fibrosis (CF) is a disease characterised by periods of clinical stability, interspersed with periods of acute deterioration, known as exacerbations. Respiratory exacerbations

\section{Strengths and limitations of this study}

This is the first long-term randomised controlled trial investigating a smartphone application for reporting symptoms in adults with cystic fibrosis.

- The pharmacist collecting primary outcome data, and the physicians prescribing intravenous antibiotics, are blinded to the participants' group allocation.

- Selecting questions to include in the smartphone application was difficult due to a lack of consensus of what defines an exacerbation in cystic fibrosis.

- It was not feasible to include spirometry in the intervention in this study which may improve clinical interpretation of reported symptoms.

have been shown to have profound and prolonged negative effects on people with $\mathrm{CF}$, such as reducing physical activity ${ }^{1}$ and health-related quality of life (HRQoL), ${ }^{2}$ increasing the rate of decline of lung function $^{3}$ and healthcare costs, ${ }^{4}$ and ultimately increasing the risk of mortality. ${ }^{5}$ The decline in lung function may also contribute to increased feelings of depression. ${ }^{6}$ In people with $\mathrm{CF}$, an exacerbation has been defined as a change in four or more disease-specific criteria comprising cough, sputum, haemoptysis, dyspnoea, fatigue, sinus pain and discharge, fever, worsening airflow obstruction (ie, reduced forced expiratory volume in $\left.1 \mathrm{~s}\left(\mathrm{FEV}_{1}\right)\right)$, anorexia or weight loss, and changes in physical examination of the chest or chest radiography. ${ }^{7}$

Of concern, of those individuals who experience an exacerbation requiring intravenous antibiotics (IVAB), approximately $25 \%$ have a persistent reduction in $\mathrm{FEV}_{1}$ at the end of their treatment, ${ }^{8}$ and more than half $(58 \%)$ of these individuals do not regain their pre-exacerbation level of $\mathrm{FEV}_{1}$ after 12 months. ${ }^{8}$ Delayed initiation of treatment has been shown to be associated with this failure to recover to baseline. ${ }^{8}$ Therefore, the late identification and treatment of an exacerbation 
due to delayed presentation will potentially worsen shortterm and long-term outcomes. People with CF may delay their presentation to a CF clinic for management of an exacerbation due to study, work or family commitments, the travel and financial burden involved in attending a CF clinic, a lack of understanding of the disease process or if affected by anxiety and depression. ${ }^{9}$

Telehealth has been evaluated in studies in people with CF using interventions such as monitoring of spirometry, ${ }^{10-12}$ oxygen saturation ${ }^{10}{ }^{13}$ and symptoms from home ${ }^{10-12}$ using videoconferencing to measure exercise capacity ${ }^{14}$ and provide health assessment in the home, ${ }^{15}$ and to provide routine outpatient clinics. ${ }^{16}$ Although telehealth, in the form of home monitoring, appears to be feasible and readily accepted by people with $\mathrm{CF},{ }^{17}$ to date, studies have not demonstrated a significant impact of telehealth on health outcomes such as exacerbation rates, lung function, HRQoL and healthcare utilisation (HCU). One reason for this may relate to the burden associated with the telehealth interventions. That is, earlier work often required participants to record measurements as often as daily and did not offer the opportunity to complete these measures outside the home (ie, there was no use of mobile technology), which compromised participant adherence to the telehealth intervention.

We aim to overcome this shortcoming by investigating the effects of using of an application for reporting symptoms that is completely mobile and stored on the participant's own smartphone. This novel approach is intended to facilitate the early identification of symptoms suggestive of a respiratory exacerbation, and allow the CF team to initiate treatment sooner in the form of oral and inhaled antibiotics, and increased mucolytic therapy and airway clearance. This may potentially reduce the risk of severe exacerbations which require IVAB and often a hospital admission.

\section{METHODS AND ANALYSIS}

This will be a prospective 12-month, single-blinded randomised controlled trial (RCT).

\section{Participant screening and selection}

Eligible participants will be identified by the principal investigator and recruited from routine outpatient clinic appointments at the adult CF centre at Sir Charles Gairdner Hospital (SCGH), Perth. The patient information and consent form will be provided to potential participants, who will then be contacted by the principal investigator approximately 48 hours later to discuss their willingness to participate.

\section{Inclusion criteria}

Adults who meet the following criteria will be eligible to participate: diagnosis of CF; aged $\geq 18$ years; under the care of the SCGH CF team; respiratory exacerbation requiring treatment with IVAB in the preceding 12 months; currently in a period of clinical stability defined as no signs of an exacerbation for the previous 2 weeks and able to understand written and spoken English.

\section{Exclusion criteria}

Criteria to exclude individuals from participating will be: previous lung transplantation or current listing for lung transplantation; inability to undertake a prescribed treatment regimen or inability to use or not being in possession of a smartphone.

\section{Randomisation and allocation concealment}

Participants will be randomly allocated to the intervention or control group using the University of Sydney National Health Medical Research Council Clinical Trials Centre randomisation service. Recruitment will be stratified according to gender, location (metropolitan vs rural and remote) and whether the participant is prescribed ivacaftor or lumacaftor/ivacaftor, as these medications can reduce the rate of exacerbations and improve lung function, weight and HRQoL. ${ }^{18} 19$

\section{Study measurements}

Participants will have assessments performed at baseline (prior to randomisation), and at 6 and 12 months, unless otherwise stated. Assessments will be performed when the participant is in a period of clinical stability, defined as no signs of an exacerbation for the previous 2 weeks. If the participant has signs of an exacerbation at their scheduled follow-up time, the assessments will be completed at the subsequent visit when they are clinically stable. The principal investigator will perform all assessments during follow-up visits at SCGH. For telehealth assessments, spirometry and weight will be assessed by a health professional local to the participant, with the remainder of the assessments completed by the principal investigator.

\section{Primary outcome}

Number of courses of IVAB and IVAB days

Information regarding the use of IVAB will be obtained from the medical records of the participants by a pharmacist blinded to the study groups and verified against SCGH pharmacy dispensing records. This will be collected at the final assessment only.

\section{Secondary outcomes}

Lung function ( $\mathrm{FEV}_{1}$ )

Lung function will be measured using a Medgraphics USB spirometer (MGC Diagnostics, Minnesota, USA) or Easyone spirometer (ndd Medical Technologies, Massachusetts, USA). Each participant will perform up to eight maximal forced expiratory manoeuvres to ensure the two best attempts meet published standards. ${ }^{20}$

\section{HRQoL: Cystic Fibrosis Questionnaire-Revised}

This self-complete questionnaire comprises 50 questions answered on a 4-point Likert scale, and covers 12 domains comprising physical, vitality, emotion, treatment burden, health, social, body, role, weight, respiratory, digestion and eating. ${ }^{21}$ The Cystic Fibrosis Questionnaire-Revised 
requires the user to recall information from the previous 2 weeks.

\section{Feelings of anxiety and depression: Hospital Anxiety and} Depression Scale

The Hospital Anxiety and Depression Scale ${ }^{22}$ contains 14 self-report questions, evenly divided into anxiety and depression scales (seven each). A 4-point Likert scale $(0-3)$ is used to answer questions based on a recall period of 7 days.

\section{Nutritional status: body mass index}

Participants will have their height and weight measured, and body mass index (BMI) will be calculated.

\section{Measures of HCU including time to first exacerbation requiring} IVAB, oral and inhaled antibiotic use and cost, hospital admissions and cost, and number of $\mathrm{CF}$ clinic visits

At the final assessment only, the participant's medical record will be reviewed to extract information pertaining to HCU. This includes: time (in days) from randomisation to the first exacerbation requiring IVAB; number of hospital admissions and days; number of courses and days of oral and inhaled antibiotics; number of CF clinic visits; estimated cost of IVAB and other antibiotic treatment; and estimated cost of hospital admissions. Costs related to HCU will be estimated using information provided from the SCGH Pharmacy and Finance departments. All potential participants receive their care at the SCGH CF centre or via shared care with an external respiratory physician (and not from a general practitioner). Prescribed antibiotic use will be well documented in the medical record, and participants will not be required to keep a diary of antibiotic use. Non-clinical costs relating to participants' travel, parking, time off work and telephone calls will not be recorded.

\section{Medication adherence: Treatment Adherence Questionnaire-CF}

Adherence to routine $\mathrm{CF}$ treatments will be assessed using the Treatment Adherence Questionnaire (TAQ$\mathrm{CF}) .^{23}$ This measure has 12 treatment items and asks the user to report on the frequency of each treatment on a 7-point Likert scale (ranging from 'not at all' to ' 3 or more times per day'), and the duration of treatment on a 6-point Likert scale (' 0 ' to ' $25+$ ' $\mathrm{min}$ ). The TAQ-CF also asks the participant to report on the barriers to individual treatments.

\section{Absenteeism/presenteeism: WHO's Health and Work Performance Questionnaire}

Absenteeism is defined as being absent from work or study due to illness whereas presenteeism is defined as the loss of productivity associated with attending work or study when unwell. ${ }^{24}$ This questionnaire uses the absenteeism and presenteeism questions of the Health and Work Performance Questionnaire. ${ }^{25}$

\section{System usability: System Usability Scale}

The System Usability Scale ${ }^{26}$ is a validated assessment tool comprising 10 questions regarding the usability of
Box 1 Smartphone application questions (participants answer yes or no)

In the past week, have you had:

Worsening sputum volume or colour?

New or increased blood in your sputum?

Increased cough?

New or increased chest pain?

New or increased wheeze?

New or increased chest tightness?

Increased shortness of breath or difficulty breathing?

Increased fatigue or lethargy?

Fever?

Loss of appetite or weight?

Sinus pain or tenderness?

In the past week do you feel that your health has worsened?

In the past week, have you felt:

Low in mood?

Worried?

technological systems, and is answered on a 5-point Likert scale. Other measures of system usability will include the time of day the smartphone application is used and how many times the questions were not completed.

\section{Intervention}

A smartphone application has been developed for use by participants to report symptoms on their own smartphones. The application contains 14 questions (box 1) that are required to be answered 'yes' or 'no'. Twelve questions relate to respiratory symptoms; 10 questions are derived from the Fuchs exacerbation scale, ${ }^{7}$ with the addition of two symptoms (wheeze and chest tightness) commonly identified by adults with $\mathrm{CF}$ as indicators of an exacerbation ${ }^{27}$ and considered important by the research team. The application also asks the participant to answer two questions relating to their feelings of anxiety and depression. The data will then be transmitted securely via password-protected email to members of the research team. This smartphone application has demonstrated high system usability in people with CF, as well as good observer agreement between CF clinicians interpreting the data. ${ }^{28}$

\section{Experimental group}

Participants will be asked to use the smartphone application once per week on a set day and time of their choosing, for a period of 12 months. A smartphone calendar alert will remind the participant to answer the symptom questions. Participants can also use the application earlier than the next weekly reporting time if they feel their symptoms have worsened. If the participant misses one of their weekly reporting times, they will be prompted to answer the application questions via text message. If the participant responds 'yes' to any of the symptom questions, the principal investigator will alert the CF nurse practitioner, who will then phone the participant to discuss their symptoms and whether treatment 


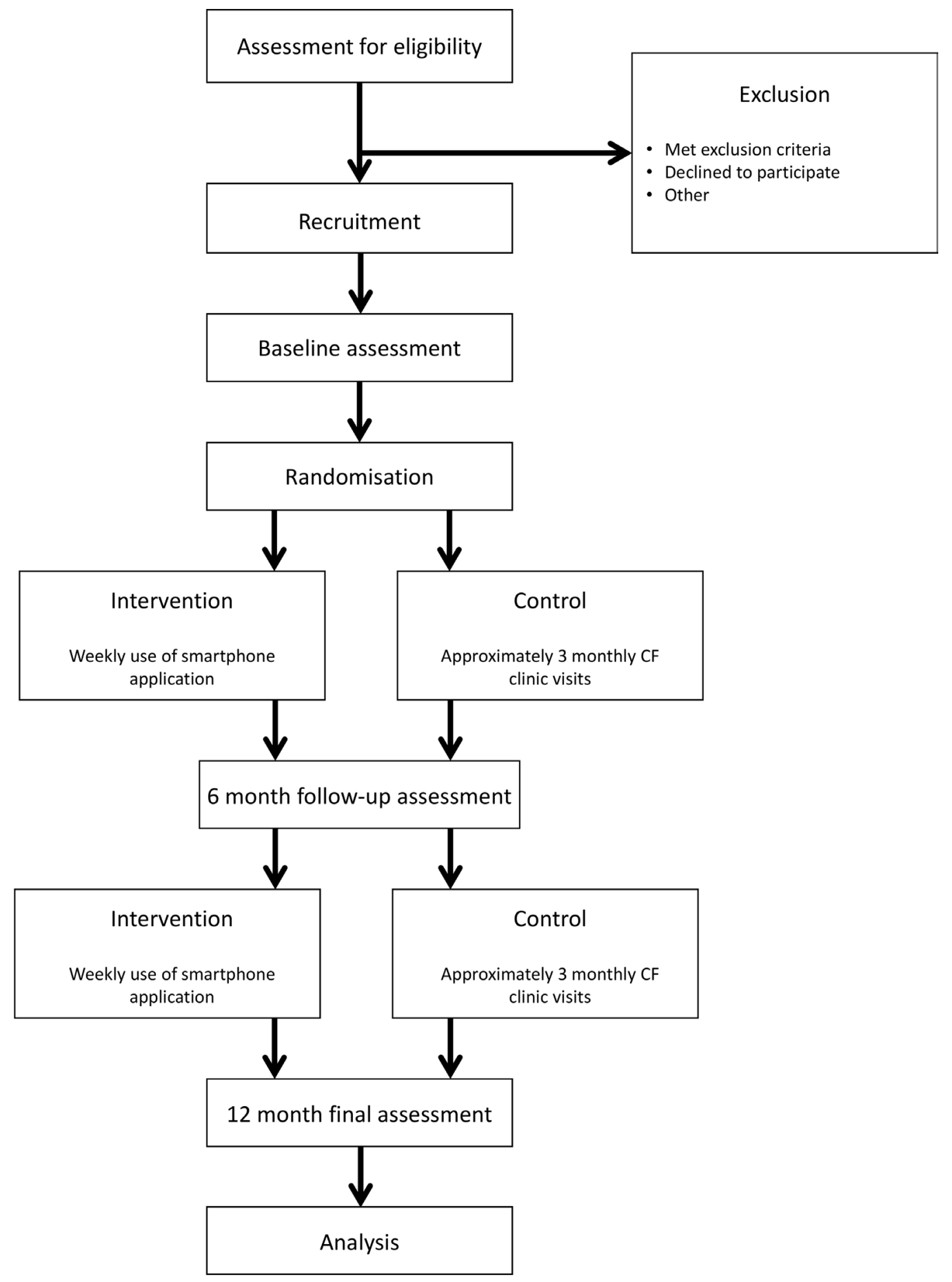

Figure 1 'Study design'. CF, cystic fibrosis.

(ie, oral or inhaled antibiotics) is required, in consultation with a CF physician blinded to the participant's study group. The CF nurse practitioner will also determine if a formal review in the CF clinic is required. If the participant's respiratory disease has been stable for 3 months, they will attend a routine CF clinic appointment.

\section{Control group}

Participants in this group will continue to receive usual care, involving routine CF clinic appointments (approximately 3 monthly). Participants can contact the CF Nurse
Practitioner via telephone sooner if there is a change in their clinical status.

\section{Statistical analysis}

Statistical analyses will be undertaken with Stata (StataCorp, Stata statistical Software Release: V.14) in accordance with the intention-to-treat principle. The distribution of data will be assessed for normality. A p value of $<0.05$ will be considered statistically significant. Negative binomial regression, and independent t-tests or Mann-Whitney $\mathrm{U}$ tests will be used to determine 
differences between the two groups. Further analyses will include general linear models to adjust for possible covariates.

\section{Number of courses of IVAB and number of IVAB days}

Summary statistics and frequencies will be provided for continuous and categorical variables. Descriptive plots will also be produced for all variables. Independent t-tests will be used to determine whether the number of courses of IVAB, and number of IVAB days, differs between the two groups. Further analyses will include general linear models to adjust for possible covariates including age, gender, BMI, Pseudomonas aeruginosa, pancreatic insufficiency and CF-related diabetes. Appropriate transformations will be conducted if the data are not normally distributed.

\section{Other measurements}

For all variables, a between-group analysis of these outcome measures will be performed using an independent t-test (parametric data) or Mann-Whitney U test (non-parametric data). Within group changes in outcome measures collected at each visit will be assessed using one-way repeated measures analysis of variance (parametric data) or Friedman's test (non-parametric data).

\section{Sample size calculations}

This is a study comparing participants who will use a smartphone application and those who will not. To investigate the difference in the number of IVAB courses between the two groups, assuming a within group SD of 1.21 (based on IVAB usage at the SCGH CF centre during the 2 years prior to the study), a sample size of 23 in each group will be required to detect a difference of one exacerbation requiring IVAB per participant, with $80 \%$ power and a significance level of $\mathrm{p}<0.05$. To account for any loss to follow-up or drop-out, we will recruit an additional seven individuals per group for a total sample size of 60 . No additional individuals are required to detect the same difference under non-parametric settings.

\section{Patient and public involvement statement}

The intervention used in this study was designed with input from participants in a pilot study conducted by the research team. ${ }^{28}$ There was no further involvement in the development of this RCT by patients or the public.

\section{DATA COLLECTION AND MANAGEMENT}

Participant data will not contain any information that allows the person's identity to be known. All data stored electronically at SCGH will be kept on a password-protected computer or locked in a filing cabinet in the Department of Respiratory Medicine. Any printed information including signed consent forms will be kept in locked filing cabinets. Data will be kept for a minimum of 15 years. After this time, data collection forms will be shredded and electronic files will be deleted.
Data transmitted from the smartphone application will be transmitted securely via password-protected email to members of the research team at SCGH, and not kept in 'cloud' storage at any stage. After transmission from the participant's phone or tablet, the data will be automatically deleted, preventing any potential breach of privacy if the device is lost or stolen. The application will also request the user to enter a unique, four-digit passcode before being able to access the application questions.

\section{ETHICS}

This trial has been registered with the Australian and New Zealand Clinical Trials Registry (ACTRN12615000599572). Results of this study will be presented at international conferences and published in peer-reviewed journals in accordance with the Consolidated Standards of Reporting Trials statement (figure 1). ${ }^{29}$

\section{DISCUSSION}

This will be the first RCT investigating the impact of a smartphone application used by people with CF to report symptoms suggestive of a respiratory exacerbation directly to the CF team. By reducing the delay often seen in the reporting of symptoms, and therefore facilitating the earlier provision of treatment, the severity of exacerbations and the need for IVAB and hospital admissions may be reduced.

Contributors JW, SJ, DP, SMu, SMo, NC and KH assisted in the design of the study protocol. JW drafted the manuscript, which was also contributed by SJ, DP, SMu, $\mathrm{SMo}, \mathrm{NC}$ and KH. All authors read and approved the final version of the manuscript.

Funding This study has received funding support from the Sir Charles Gairdner Group Research Advisory Council Grant (2015-16/040). This work has also received in-kind funding from the Sir Charles Gairdner Hospital Physiotherapy Department and Department of Respiratory Medicine and Curtin University.

Competing interests JW reports grants from Sir Charles Gairdner Group Research Advisory Council, during the conduct of the study, grants from Technipro Pulmomed, non-financial support from Novartis, non-financial support from Pharmaxis, outside the submitted work. SJ reports grants from Sir Charles Gairdner Group Research Advisory Council, during the conduct of the study. SMu reports grants from Sir Charles Gairdner Group Research Advisory Council, during the conduct of the study, personal fees from Vertex, non-financial support from Novartis, outside the submitted work.

Patient consent Not required.

Ethics approval Approval was obtained from the Sir Charles Gairdner Group Human Research Ethics Committee for WA Health (2015-2030) and Curtin University Human Research Ethics Committee (HR212/2015).

Provenance and peer review Not commissioned; externally peer reviewed.

Open Access This is an Open Access article distributed in accordance with the Creative Commons Attribution Non Commercial (CC BY-NC 4.0) license, which permits others to distribute, remix, adapt, build upon this work non-commercially, and license their derivative works on different terms, provided the original work is properly cited and the use is non-commercial. See: http://creativecommons.org/ licenses/by-nc/4.0/

(C) Article author(s) (or their employer(s) unless otherwise stated in the text of the article) 2018. All rights reserved. No commercial use is permitted unless otherwise expressly granted. 


\section{REFERENCES}

1. Ward N, White D, Rowe $\mathrm{H}$, et al. Physical activity levels of patients with cystic fibrosis hospitalised with an acute respiratory exacerbation. Respir Med 2013;107:1014-20.

2. Britto MT, Kotagal UR, Hornung RW, et al. Impact of recent pulmonary exacerbations on quality of life in patients with cystic fibrosis. Chest 2002;121:64-72.

3. Sanders DB, Bittner RC, Rosenfeld M, et al. Pulmonary exacerbations are associated with subsequent FEV1 decline in both adults and children with cystic fibrosis. Pediatr Pulmonol 2011;46:393-400.

4. Robson M, Abbott J, Webb K, et al. A cost description of an adult cystic fibrosis unit and cost analyses of different categories of patients. Thorax 1992;47:684-9.

5. de Boer K, Vandemheen $\mathrm{KL}$, Tullis $\mathrm{E}$, et al. Exacerbation frequency and clinical outcomes in adult patients with cystic fibrosis. Thorax 2011;66:680-5.

6. Riekert KA, Bartlett SJ, Boyle MP, et al. The association between depression, lung function, and health-related quality of life among adults with cystic fibrosis. Chest 2007;132:231-7.

7. Fuchs HJ, Borowitz DS, Christiansen DH, et al. Effect of aerosolized recombinant human DNase on exacerbations of respiratory symptoms and on pulmonary function in patients with cystic fibrosis. The Pulmozyme Study Group. N Engl J Med 1994;331:637-42.

8. Sanders DB, Bittner RC, Rosenfeld M, et al. Failure to recover to baseline pulmonary function after cystic fibrosis pulmonary exacerbation. Am J Respir Crit Care Med 2010;182:627-32.

9. Zemanick ET, Harris JK, Conway S, et al. Measuring and improving respiratory outcomes in cystic fibrosis lung disease: opportunities and challenges to therapy. J Cyst Fibros 2010;9:1-16.

10. Grzincich G, Gagliardini R, Bossi A, et al. Evaluation of a home telemonitoring service for adult patients with cystic fibrosis: a pilot study. J Telemed Telecare 2010;16:359-62.

11. Jarad NA, Sund ZM. Telemonitoring in chronic obstructive airway disease and adult patients with cystic fibrosis. J Telemed Telecare 2011;17:127-32.

12. Lechtzin N, Mayer-Hamblett N, West NE, et al. Home monitoring of patients with cystic fibrosis to identify and treat acute pulmonary exacerbations. elCE Study Results. Am J Respir Crit Care Med 2017;196:1144-51.

13. Bella S, Murgia F, Tozzi AE, et al. Five years of telemedicine in cystic fibrosis disease. Clin Ter 2009;160:457-60.
14. Cox NS, Alison JA, Button BM, et al. Assessing exercise capacity using telehealth: a feasibility study in adults with cystic fibrosis. Respir Care 2013;58:286-90.

15. Wilkinson OM, Duncan-Skingle F, Pryor JA, et al. A feasibility study of home telemedicine for patients with cystic fibrosis awaiting transplantation. J Telemed Telecare 2008;14:182-5.

16. Wood J, Mulrennan S, Hill K, et al. Telehealth clinics increase access to care for adults with cystic fibrosis living in rural and remote Western Australia. J Telemed Telecare 2017;23.

17. Cox NS, Alison JA, Rasekaba T, et al. Telehealth in cystic fibrosis: a systematic review. J Telemed Telecare 2012;18:72-8.

18. Ramsey BW, Davies J, McElvaney NG, et al. A CFTR potentiator in patients with cystic fibrosis and the G551D mutation. N Engl J Med 2011;365:1663-72.

19. Wainwright CE, Elborn JS, Ramsey BW. Lumacaftor-ivacaftor in patients with cystic fibrosis homozygous for phe508del CFTR. N Engl J Med 2015;373:220-31.

20. Miller MR, Hankinson J, Brusasco V, et al. Standardisation of spirometry. Eur Respir J 2005;26:319-38.

21. Quittner AL, Buu A, Messer MA, et al. Development and validation of The Cystic Fibrosis Questionnaire in the United States: a health-related quality-of-life measure for cystic fibrosis. Chest 2005;128:2347-54.

22. Zigmond AS, Snaith RP. The hospital anxiety and depression scale. Acta Psychiatr Scand 1983;67:361-70.

23. Quittner AL, Modi AC, Lemanek KL, et al. Evidence-based assessment of adherence to medical treatments in pediatric psychology. J Pediatr Psychol 2008;33:916-36. discussion 37-8.

24. Wang PS, Beck A, Berglund P, et al. Chronic medical conditions and work performance in the health and work performance questionnaire calibration surveys. J Occup Environ Med 2003;45:1303-11.

25. Kessler RC, Barber C, Beck A, et al. The world health organization health and work performance questionnaire (HPQ). J Occup Environ Med 2003;45:156-74.

26. Brooke J. SUS: A "quick and dirty" usability scale. In: Jordan BT PW, Weerdmeester BA, McClelland AL, eds. Usability evaluation in industry. London: Taylor and Francis, 1996:189-94.

27. Goss CH, Edwards TC, Ramsey BW, et al. Patient-reported respiratory symptoms in cystic fibrosis. J Cyst Fibros 2009;8:245-52.

28. Wood J, Jenkins S, Putrino D, et al. High usability of a smartphone application for reporting symptoms in adults with cystic fibrosis. $J$ Telemed Telecare 2017;1357633X:1357633X1772336.

29. Schulz KF, Altman DG, Moher D, et al. CONSORT 2010 statement: updated guidelines for reporting parallel group randomised trials. BMJ 2010;340:c332. 